\title{
Pigeons' memory for time: Assessment of the role of subjective shortening in the duration-comparison procedure
}

\author{
Patrick Van Rooyen and Angelo Santi \\ Wilfrid Laurier University, Waterloo, Ontario, Canada
}

\begin{abstract}
Pigeons were trained in a duration-comparison procedure to peck one key if the comparison duration (c) was $1 \mathrm{sec}$ shorter than a standard duration $(s)$, and another key if $c$ was $1 \mathrm{sec}$ longer than $s$. During training, the $s-c$ delay was $1 \mathrm{sec}$, and the total duration of an $s-c$ pair was not predictive of the correct choice. In Experiment 1 , during equal-duration pair test trials, pigeons increasingly responded long (i.e., $c>s$ ) as the $s-c$ delay was lengthened. In Experiment 2, we demonstrated that $s$ affected long responding on equal-duration test trials, even at the 8 -sec $s-c$ delay. In Experiment 3, long responding increased as the $s-c$ delay was lengthened, even when stimulus conditions during the $s-c$ delay differed from those during the intertrial interval (ITI). Additional analyses indicated that it was unlikely that the increase in long responding was due to the pigeons' adding the $s-c$ delay to $c$ and comparing the total against the duration of $s$. The increase in long responding with an increase in $s-c$ delay is more consistent with subjective shortening of $s$ than with confusion between the $s-c$ delay and the ITI.
\end{abstract}

Pigeons' memory for time is typically studied using a symbolic delayed matching-to-sample (SDMTS) procedure whereby a pigeon is required to indicate which one of two possible sample durations was presented on a given trial. The sample durations typically consist of the presentation of either a short-sample (e.g., 2-sec) or a longsample (e.g., 8-sec) stimulus, such as illumination of the houselight or the feederlight. Following trials initiated by the short sample, a pigeon may be required to peck a green key for reinforcement, and, following trials initiated by the long sample, to peck a red key for reinforcement. Retention functions for these sample durations often exhibit a response bias known as the choose-short effect (Kraemer, Mazmanian, \& Roberts, 1985; Sherburne, Zentall, \& Kaiser, 1998; Spetch, 1987; Spetch \& Wilkie, 1983). The choose-short effect is characterized by above-chance accuracy on short-sample trials as a delay interval (DI) is placed between the end of the sample presentation period and the opportunity to respond. Accuracy on the longsample trials, on the other hand, decreases to or below chance the longer the DI between the end of the sample presentation period and the opportunity to respond. The subjective shortening hypothesis (Spetch \& Wilkie, 1983) proposes that the representation of the long sample in working memory shortens and increasingly becomes more similar to the representation of the short sample in working memory as the DI increases.

More recent studies, however, have provided evidence that the choose-short effect may arise from methodological artifacts of the SDMTS procedure rather than from temporal foreshortening (Dorrance, Kaiser, \& Zentall, 2000;
Sherburne et al., 1998; Zentall, 1997, 1999, 2007). The instructional ambiguity/confusion hypothesis explains the choose-short effect in terms of a confusion that can occur when the DI and intertrial interval (ITI) share similar ambient illumination conditions (e.g., the houselight is either on or off during both the ITI and DI). If pigeons confuse the DI with the ITI, when presented with choice stimuli at the end of a DI, they may respond as if no sample had been presented on that trial. Because the lack of a sample is more similar to a short sample than to a long sample, pigeons would be biased to respond to the stimulus correct for the short sample. However, when the ambient illumination condition differs between the DI and ITI, accuracy for both samples declines equally as the length of the DI is increased (Sherburne et al., 1998).

Time perception in pigeons has also been investigated with a duration-comparison procedure (Dreyfus, Fetterman, Smith, \& Stubbs, 1988; Dreyfus, Fetterman, Stubbs, \& Montello, 1992; Fetterman, 1987, 2006; Fetterman \& Dreyfus, 1986; Fetterman, Dreyfus, Smith, \& Stubbs, 1988; Fetterman, Dreyfus, \& Stubbs, 1989; Kraemer, 1990). In the duration-comparison procedure, rather than being presented with a single-sample duration, as in the SDMTS procedure, a pigeon is presented with a standard duration $(s)$ followed by a comparison duration $(c)$, and the pigeon must peck one key if $c<s$, and another if $c>s$. Because the duration-comparison procedure requires the subject to make a relational decision between $s$ and $c$, it naturally involves memory, even if the subject is trained with a $0-\sec s-c$ delay. Although the majority of duration-comparison studies have examined the ability of

A.Santi, asanti@wlu.ca 
pigeons to make relational discriminations (Dreyfus et al., 1988; Dreyfus et al., 1992; Fetterman, 1987; Fetterman et al., 1989; Kraemer, 1990), a few have tested pigeons at increasing $s-c$ delays. Fetterman and Dreyfus found that pigeons were more likely to make a long response (i.e., indicate $c>s$ ) when tested with equal-duration pairs at a 0 -sec $s-c$ delay. It was also found that when tested with various unequal-duration pairs at $s-c$ delays of up to $30 \mathrm{sec}$, pigeons increasingly made long responses. Furthermore, Fetterman et al. (1988) found similar results when pigeons were trained with a $0-\sec s-c$ delay and were then subsequently tested with extended $s-c$ delays. In both studies, the pigeons were trained with the houselight on during the sample presentation phase as well as during the $s-c$ delay, but not during the ITI. Thus, pigeons should not have confused the ITI with the $s-c$ delay. Fetterman et al. (1989) suggested that the subjective shortening of $s$ over the $s-c$ delay may have been responsible for the increase in long responding.

One of the limitations of the duration-comparison procedure is that for duration pairs with extreme values of $s$ or $c$, decisions can be made solely on the basis of $s$ or $c$ alone rather than on the relation of $c$ to $s$. This can create problems for data interpretation. In Fetterman et al.'s (1988) study, pigeons were trained with all possible combinations of duration pairs, which ranged from 0.5 to $16.0 \mathrm{sec}$. When the comparison duration belonged to an extreme category (i.e., 0.5-2 sec; 8.5-16 sec), pigeons' accuracy remained above chance even at the longest $s-c$ delays. However, when the comparison duration belonged to an intermediate duration (i.e., 2.5-4.0 sec; 4.5-8.0 sec), pigeons' accuracy was closer to chance performance as the $s-c$ delay increased. Thus, if a pigeon can respond on the basis of the absolute duration of $c$ alone, then memory of $s$ is not being tested. In addition, regardless of whether there is a delay interposed between $s$ and $c$, the longer $c$ is, the more likely that $s$ will be forgotten and a $c>s$ response would be made (Fetterman \& Dreyfus, 1986; Fetterman et al., 1988).

Experiments investigating humans' memory for time in the duration-comparison procedure have a longer history and have been focused more on the phenomenon of explaining time-order errors (TOEs; Allan, 1977, 1979; Hellström \& Rammsayer, 2004; Jamieson, 1977; Jamieson \& Petrusic, 1975a, 1975b, 1976; Stott, 1935; Woodrow, 1935; Woodrow \& Stott, 1936). In contrast to research with pigeons, the human studies have employed duration ranges on the order of milliseconds to prevent chronometric counting and duration pairs that prevent the identification of the correct response on the basis of the absolute duration of $s$ or $c$ alone. In a study investigating subjective shortening in humans, Wearden and Ferrara (1993) asked participants to indicate whether $c$ was the same as or different from $s$. They found that duration pairs of equal length (e.g., $0.5 \mathrm{sec}$ followed by $0.5 \mathrm{sec}$ ) were more likely to be judged different in duration as the $s-c$ delay increased. This difference suggested that the representations of $s$ and $c$ in working memory were not stable over time. In another experiment, participants were given the opportunity to report whether $c$ was shorter than, longer than, or equal to $s$, and again, the likelihood of reporting that $c$ was longer than $s$ increased as a function of the $s-c$ delay. Wearden and Ferrara suggested that this increase in long responding (i.e., selecting the response alternative designated as correct for $c>s$ trials) occurred because of the subjective shortening of $s$. Wearden, Goodson, and Foran (2007) also found evidence for subjective shortening when they tested subjects with filled and unfilled auditory and visual intervals that ranged from 250 to $650 \mathrm{msec}$. Filled intervals consisted of the presentation of continuous tones or continuous visual stimuli, whereas unfilled intervals consisted of the presentation of very brief clicks or lines. Subjects were required to indicate whether $c$ was $150 \mathrm{msec}$ shorter than $s, 150 \mathrm{msec}$ longer than $s$, or equal to $s$. For equal-duration pairs, the number of long responses increased as a function of $s-c$ delay length for filled and unfilled visual stimuli and filled auditory stimuli, but not for unfilled auditory stimuli. In a second experiment, subjects were given the opportunity to respond only that $c$ was longer or shorter than $s$ and, once again, the number of long responses on equal-duration pair test trials increased as a function of $s-c$ delay length for all trial types. Although subjective shortening has been suggested to be responsible for the increase in long responding observed in human experiments, whether the same can be said about experiments in nonhumans has yet to be adequately investigated.

The purpose of the present series of experiments was to assess the role of subjective shortening in the durationcomparison task and to attempt to answer why pigeons increasingly select the response alternative correct for $c>s$ trials as the $s-c$ delay increases. In Experiment 1, pigeons were trained and tested with $s-c$ delays that shared similar ambient illumination conditions with the ITI (i.e., could be confused with the ITI). In Experiment 2, probe test trials in which only the $c$ duration was presented were used to investigate whether the long responding in Experiment 1 at the 8-sec $s-c$ delay was due to pigeons' confusing the $s-c$ delay with the ITI and therefore responding solely on the basis of $c$, or whether-consistent with subjective shortening - there was evidence that responding at the 8 -sec $s-c$ delay was affected by a memory of $s$. In Experiment 3 , the stimulus conditions during the $s-c$ delay were varied so that they were either similar to (i.e., confusable with) or different from (i.e., not confusable with) the ITI. Doing this provided a more direct assessment of whether confusion plays any role in the increase in long responding observed as the $s-c$ delay increases.

\section{EXPERIMENT 1}

The goal of the first experiment was to examine the role of subjective shortening in the duration-comparison task when the $s-c$ delay and the ITI shared similar illumination conditions. Although subjective shortening has been proposed to account for the increase in long responding observed in humans (Wearden \& Ferrara, 1993; Wearden et al., 2007; Wearden, Parry, \& Stamp, 2002), the process responsible for the increase in long responding observed in pigeons has remained somewhat unclear. Although 
Fetterman et al. (1988) trained pigeons with a distinctive trial presentation phase (i.e., houselight illumination) that differentiated the $s-c$ delay (i.e., the interval between the offset of $s$ and the onset of $c$ ) from the ITI and prevented confusion, it was possible that in their study, the increase in long responding occurred because pigeons added the $s-c$ delay to $c$ and then compared the total against the duration of $s$. In order to minimize this potential problem in the present study, the duration pairs were constructed so that pigeons would be unable to completely rely on the absolute duration of either $s$ or $c$ or on the total length of a trial. Thus, the main objectives of Experiment 1 were to observe whether pigeons demonstrate an increase in long responding as a function of $s-c$ delay length when the total duration of $s$ and $c$ was controlled for, and to elucidate whether an increase in long responding at extended $s-c$ delays also occurs when the $s-c$ delay and ITI share similar ambient illumination conditions.

\section{Method}

\section{Subjects}

Eight adult Silver-King pigeons were individually housed and maintained at $80 \%$ of their adult free-feeding body weights. Constant access to grit and fresh water was provided, and the subjects were given postsession feedings of Purina Pigeon Chow to maintain their reduced body weights. The colony room was kept on a 12:12-h light:dark schedule, with the lights turning on at 7:00 a.m. each day. Testing was conducted 5 days per week between 8:00 a.m. and 1:00 p.m. Five of the 8 birds (Nos. 34, 35, 37, 13, and 22) had previously served in a symbol summation study (Olthof \& Santi, 2007), and the 3 remaining birds (Nos. 14, 15, and 18) had previous experience in a study examining the perception of filled and empty intervals (Santi, Keough, Gagne, \& Van Rooyen, 2007). Although the previous experience of the subjects differed, discrimination accuracy on the duration-comparison task was equivalent in both groups of pigeons at the end of training.

\section{Apparatus}

Four Coulbourn Instruments (Allentown, PA) modular operant test chambers (Model E10-10), each housed within individual isolation cubicles (Model E10-20), were used. Each cubicle was equipped with a baffled air-intake system and ventilation fan. Each test chamber was equipped with three circular translucent keys that were horizontally aligned approximately at a pigeon's standing sight line. Behind each of the keys was a projector that displayed red, green, and yellow onto a frosted rear projector screen (Model E21-18). Directly below the center key was a $5.7 \times 5 \mathrm{~cm}$ opening, which (during reinforcement) provided access to a food hopper containing mixed grain. During reinforcement, a light inside the opening was illuminated (Model E14-10 with bulb S11819X). Located $6.5 \mathrm{~cm}$ above the center key was a houselight (Model E14-10) that was capable of directing light upward to reflect off of the top of the cage, but this was not used in the present series of experiments. The organization and recording of all experimental events and responses were performed by a microcomputer system within the same room.

\section{Procedure}

Discrimination training. Figure 1 illustrates the durationcomparison procedure used in the present experiment. A trial began with the presentation of $s$, which consisted of a red light presented on all three keys. Because trials were not initiated by a keypeck, stimuli were presented on all three keys in order to enhance attention to the presentation of $s$. Following the presentation of $s$, there was a 1-sec $s-c$ delay during which all three of the keys were dark. At the end of the 1-sec dark $s-c$ delay, $c$ was presented, which consisted of a green light presented on all three keys. Following the presentation of $c$, all

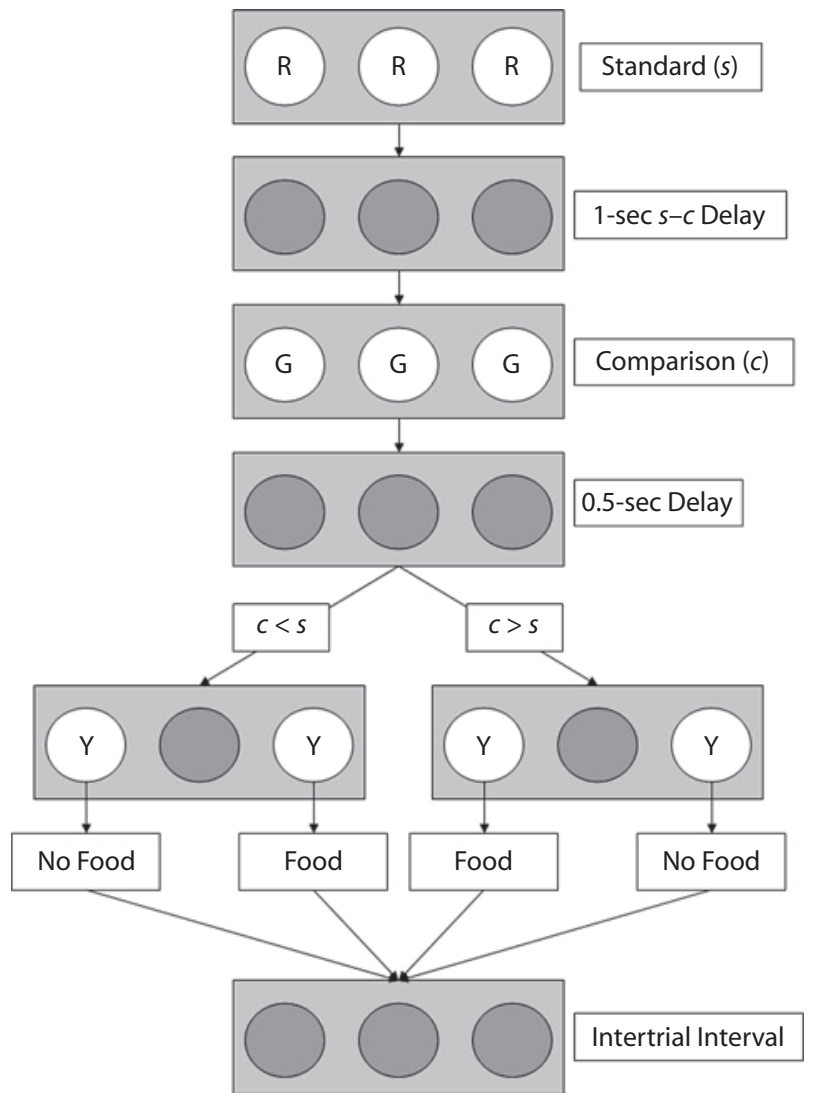

Figure 1. An illustration of the duration-comparison procedure used in Experiment 1.

three keys were darkened for $0.5 \mathrm{sec}$; then, a yellow light was presented on the left and the right keys. The short 0.5 -sec delay between $c$ and the presentation of yellow side keys was used to minimize accidental response choice due to possible perseverative pecks during the presentation of $c$. For half of the pigeons, if $c<s$, a peck to the right key was correct, whereas if $c>s$, a peck to the left key was correct. Assignment of the correct response for the remaining pigeons was reversed. Correct responses were reinforced by $4-\mathrm{sec}$ access to mixed grain, whereas incorrect responses resulted in a 4-sec time-out and a re-presentation of the same duration pair (i.e., a correction trial). The correction trial was repeated until a correct response was made. Only the choice response on the initial (noncorrection) trial was used in data analysis. Following food reward, a dark ITI of variable length $(4,8,16$, or $32 \mathrm{sec})$ was presented. Within each block of 10 trials, each of the five different duration pairs for $c<s$ trials and the five different duration pairs for $c>s$ trials occurred once in an individually randomized order. Each session consisted of 160 trials. Pigeons were trained on the duration-comparison task for 75 sessions, and then $s-c$ delay testing began. On the 75 th session of discrimination training, the mean percent correct was $88.0 \%$ on $c<s$ trials and $68.4 \%$ on $c>s$ trials. This difference in accuracy indicates that the pigeons were somewhat biased to make a $c<s$ response. In general, this bias reflected the tendency across discrimination training sessions for $c<s$ responses to increase on $c<s$ trials more than $c>s$ responses increased on $c>s$ trials.

Duration values. Table 1 lists the 10 duration pairs used during $1-\sec s-c$ delay training. The construction of duration pairs used in the present study was modeled on those used in previous studies conducted with humans by Wearden and colleagues (Wearden \& Ferrara, 1993; Wearden et al., 2007; Wearden et al., 2002). The range of the durations used was $1.0-4.0 \mathrm{sec}$ in 0.5 -sec increments. On $c<s$ trials, 
Iable 1

Values Used (in Seconds) for the Standard Durations (s), Comparison Durations (c), and Total Durations (td) Across Comparison Shorter and Comparison Longer Trials During Duration Discrimination Training

\begin{tabular}{|c|c|c|c|c|c|}
\hline \multicolumn{3}{|c|}{$\begin{array}{c}\text { Comparison } \\
\text { Shorter Trials } \\
(c \text { is } 1 \mathrm{sec} \text { shorter than } s)\end{array}$} & \multicolumn{3}{|c|}{$\begin{array}{c}\text { Comparison } \\
\text { Longer Trials } \\
(c \text { is } 1 \mathrm{sec} \text { longer than } s)\end{array}$} \\
\hline$s$ & $c$ & $\overline{t d}$ & $s$ & $c$ & $t d$ \\
\hline & & & 1.0 & 2.0 & 3.0 \\
\hline & & & 1.5 & 2.5 & 4.0 \\
\hline 2.0 & 1.0 & 3.0 & 2.0 & 3.0 & 5.0 \\
\hline 2.5 & 1.5 & 4.0 & 2.5 & 3.5 & 6.0 \\
\hline 3.0 & 2.0 & 5.0 & 3.0 & 4.0 & 7.0 \\
\hline 3.5 & 2.5 & 6.0 & & & \\
\hline 4.0 & 3.0 & 7.0 & & & \\
\hline
\end{tabular}

Note-In the $c$ columns, comparison-common durations are denoted by bold numbers, and comparison-unique trials are denoted by italicized numbers.

$s$ ranged from 2.0 to $4.0 \mathrm{sec}$ in $0.5-\mathrm{sec}$ increments, and $c$ was $1 \mathrm{sec}$ shorter. On $c>s$ trials, $s$ ranged from 1.0 to $3.0 \mathrm{sec}$ in 0.5 -sec increments, and $c$ was $1 \mathrm{sec}$ longer. Because $c$ was always $1 \mathrm{sec}$ shorter or longer than $s$ regardless of the absolute duration of $s$, according to and consistent with Weber's law, as the duration of $s$ increased, the discrimination became more difficult. This was also the case in the previous research conducted by Wearden and colleagues (Wearden \& Ferrara, 1993; Wearden et al., 2007; Wearden et al., 2002), in which $c$ was always longer or shorter than $s$ by a constant duration. As can be seen in Table 1, the configuration of $c\langle s$ and $c\rangle s$ trials prevented pigeons from relying on the total duration of $s$ and $c$. The total duration of a given trial including $s, c$, and the 1-sec $s-c$ delay ranged from 4.0 to $8.0 \mathrm{sec}$ in $1.0-\mathrm{sec}$ increments for both $c<s$ and $c>s$ trials. Therefore, the total duration of an individual trial could not serve as a predictive cue for responding during either training or extended $s-c$ delay tests. However, there were 4 duration pairs with unique comparison durations, which made it possible for pigeons to respond correctly on the basis of the duration of $c$ alone. These 4 pairs (which will be referred to as comparison-unique trials) consisted of two $c<s$ trials $(2.0-1.0 \mathrm{sec}$ and $2.5-1.5 \mathrm{sec})$ and two $c>s$ trials
$(2.5-3.5 \mathrm{sec}$ and $3.0-4.0 \mathrm{sec})$. The 6 remaining duration pairs, which shared a common duration of $c(2.0,2.5$, and $3.0 \mathrm{sec})$, prevented pigeons from responding solely on the basis of $c$ alone. These six trials will be referred to as comparison-common trials. Duration pairs used for equal-duration pair probe testing were sampled from the entire $1.0-4.0$-sec range and were presented in increments of $0.5 \mathrm{sec}$, for a total of 7 equal-duration pairs.

Probe testing with equal-duration pairs. Probe testing with equal-duration pairs was conducted for 20 sessions, each consisting of 168 trials. Within each test session, 70 trials occurred at the 1 -sec baseline $s-c$ delay for both $c<s$ and $c>s$ duration pairs (i.e., a total of 140 baseline trials identical to training trials). Within each 6 -trial block, there were 5 baseline trials and 1 equal-duration probe trial. Equal-duration probe trials consisted of every equal-duration pair in increments of $0.5 \mathrm{sec}$ from 1.0 to $4.0 \mathrm{sec}$ occurring at the baseline $1-\sec s-c$ delay as well as at three extended $s-c$ delays $(2,4$, and $8 \mathrm{sec}$ ), for a total of 28 probe trials per session. The $s-c$ delay was always spent in darkness. Correct responses continued to be reinforced on baseline $c<s$ and $c>s$ trials. On equal-duration pair probe trials, responses to either key led directly to a variable-length dark ITI of $4,8,16$, or $32 \mathrm{sec}$. The correction procedure remained in effect on baseline $c<s$ and $c>s$ trials. All statistical analyses used a rejection region of $p<.05$.

\section{Results and Discussion}

Figure 2 illustrates the mean percentage of long responding (i.e., selecting the response alternative designated as correct for $c>s$ trials) during acquisition training across blocks of five sessions. The data are presented as a function of factorial combination of trial type $(c<s$, $c>s$ ) and comparison type (unique, common). Throughout discrimination training, pigeons were more accurate on comparison-unique trials than on comparison-common trials for both $c<s$ and $c>s$ duration pairs. This is not surprising, since the duration of $c$ alone was predictive of correct choice for comparison-unique trials but not for comparison-common trials. An ANOVA was performed on the discrimination training data with trial type $(c<s$, $c>s$ ), comparison duration type (unique, common), and

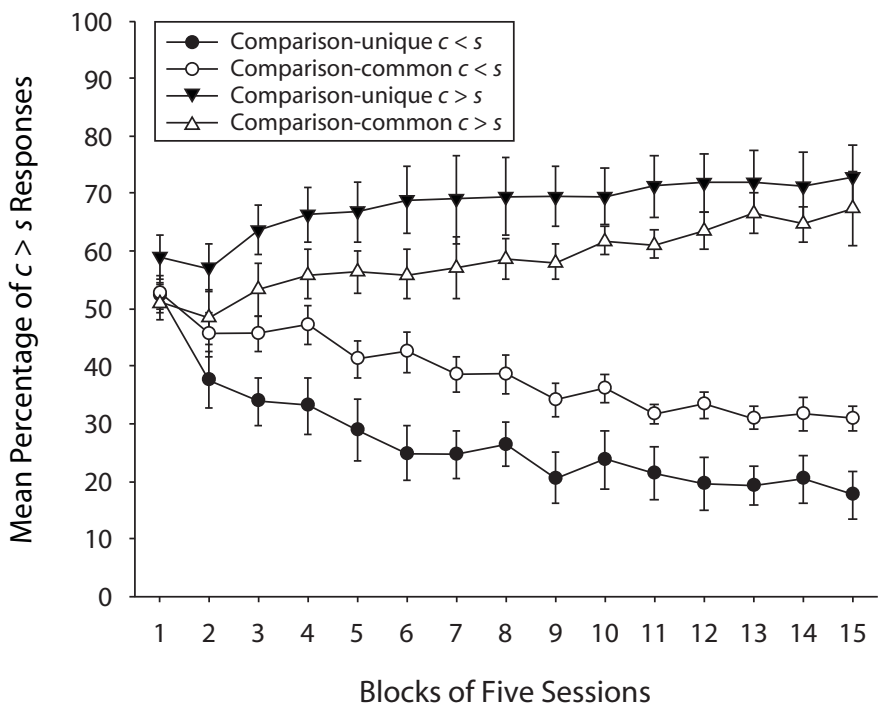

Figure 2. The mean percentage of long $(c>s)$ responding during discrimination training across blocks of five sessions for each of the trial $(c<s, c>s)$ and comparison (common, unique) types. Error bars represent the standard errors of the means. 
session blocks as within-subjects factors. The main effect of trial type indicated that pigeons made significantly more long responses on $c>s$ trials than on $c<s$ trials $[F(1,7)=60.92]$. There was also a significant trial type $\times$ comparison type interaction $[F(1,7)=9.97]$. A simple main effects analysis revealed that the percentage of long responses was significantly higher on $c>s$ unique trials than on $c>s$ common trials $[F(1,7)=7.85]$. In addition, the percentage of long responses was significantly lower on $c<s$ unique trials than on $c<s$ common trials $[F(1,7)=9.84]$. Thus, although pigeons could better discriminate between $c<s$ and $c>s$ trials when the absolute duration of $c$ provided a predictive cue, they were nevertheless able to make the discrimination even when this cue was not predictive (i.e., on comparison-common trials). The analysis also revealed a significant trial type $\times$ block interaction $[F(14,98)=16.78]$. Discrimination training resulted in a greater decrease in long responding on $c<s$ trials $[F(14,98)=15.65]$ than an increase in long responding on $c>s$ trials $[F(14,98)=2.29, p<.05]$. Overall, the analysis indicates that accuracy on both $c<s$ and $c>s$ trial types significantly increased during training and that accuracy was higher for comparison-unique trials than for comparison-common trials. During the last block of duration discrimination training sessions, mean percentcorrect responding was $82.3 \%$ for $c<s$ comparisonunique trials, $72.9 \%$ for $c>s$ comparison-unique trials, $69.1 \%$ for $c<s$ comparison-common trials, and $67.3 \%$ for $c>s$ comparison-common trials.

The mean percentage of long responding on equalduration probe trials plotted against $s-c$ delay for $c<s$ comparison-unique durations (i.e., $1.0 \mathrm{sec}, 1.5 \mathrm{sec}$ ), for comparison-common durations (i.e., $2.0 \mathrm{sec}, 2.5 \mathrm{sec}$, $3.0 \mathrm{sec}$ ), and for $c>s$ comparison-unique durations (i.e., $3.5 \mathrm{sec}, 4.0 \mathrm{sec}$ ) are shown in Figure 3. Similar to the results of previous research with pigeons (Fetterman et al.,

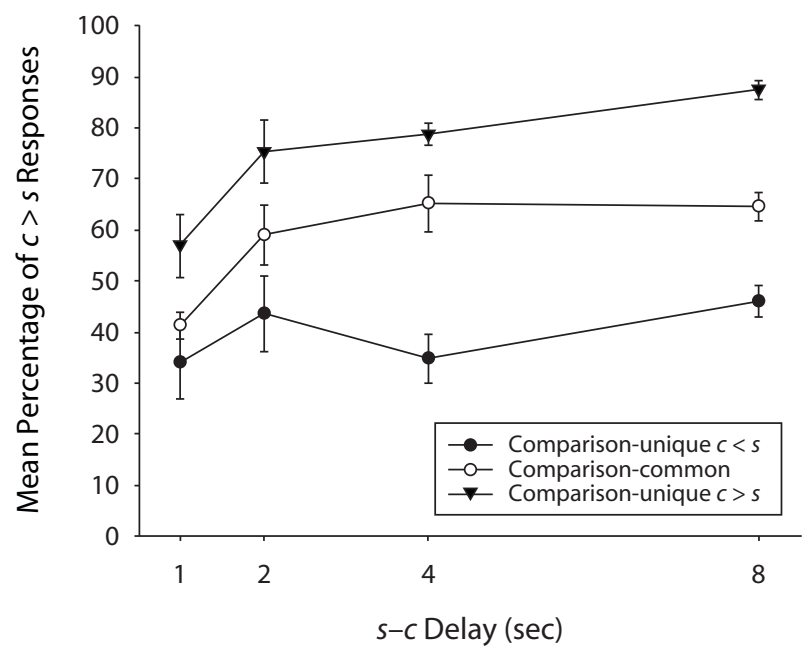

Figure 3. The mean percentage of long $(c>s)$ responding during $s-c$ delay testing with equal-duration pairs for the three different types of comparison durations: $c<s$ unique (1.0 $\mathrm{sec}$, $1.5 \mathrm{sec})$, comparison-common ( $2.0 \mathrm{sec}, 2.5 \mathrm{sec}, 3.0 \mathrm{sec})$, and $c>s$ unique (3.5 sec, $4.0 \mathrm{sec})$. Error bars represent the standard errors of the means.
1988) and humans (Wearden et al., 2007) in the durationcomparison procedure, long responding increased as a function of the $s-c$ delay length. An ANOVA was conducted on the $s-c$ delay testing data with comparison type ( $c<s$ unique, comparison-common, $c>s$ unique) and $s-c$ delay as within-subjects factors. The data revealed a significant main effect of comparison type $[F(2,14)=$ 29.97], of $s-c$ delay $[F(3,21)=14.37]$, and a significant comparison type $\times s-c$ delay interaction $[F(6,42)=2.42]$. A simple main effects analysis revealed that long responding significantly increased as a function of $s-c$ delay length on comparison-common $[F(3,21)=10.46]$ and $c>s$ unique $[F(3,21)=10.84]$ trials, but not on $c<s$ unique $[F(3,21)=1.93]$ trials. Single-sample $t$ tests were used to assess whether the mean percentage of long responses was significantly below or above $50 \%$, indicative of a $+\mathrm{TOE}$ or a - TOE, respectively. A + TOE is one in which $s$ is "weighted" more than $c$, leading to "short" responses. A -TOE is one in which $c$ is "weighted" more than $s$, leading to "long" responses. On $c<s$ unique trials, a + TOE was observed at the $1-$ and 4-sec $s-c$ delays $[t(7)=2.19$ and 3.22, respectively]. On $c>s$ unique trials, a - TOE occurred at all of the $s-c$ delays, except at the 1-sec delay $[t(7)=4.09,13.28,19.84]$. On comparison-common trials, although a + TOE was observed at the $1-\sec s-c$ delay $[t(7)=3.40]$, a - TOE was observed at the $4-$ and 8 -sec $s-c$ delays $[t(7)=2.73$ and 5.06, respectively].

The present experiment presents evidence that long responding was influenced by both the length of the comparison duration and the length of the $s-c$ delay. Although the increase in long responding with increases in $s-c$ delay might lead one to suggest that pigeons may have been comparing $s$ with the total duration of the $s-c$ delay and $c$, additional analyses of the data are inconsistent with this possibility. For example, if pigeons added the $s-c$ delay to $c$ (i.e., timing from the offset of $s$ to the offset of $c$ ), then for a baseline duration pair, such as $1.0-2.0 \mathrm{sec}$, the actual duration being compared would be 1.0-3.0 sec (i.e., 1-sec $s-c$ delay $+2.0-\sec c$ ). During $s-c$ delay testing, when presented with a $1.0-1.0$-sec equal-duration pair at a $2-\sec s-c$ delay, pigeons who added the $s-c$ to $c$ would also be comparing a 1.0-3.0-sec pair (i.e., 2-sec $s-c$ delay $+1.0-\sec c)$. Thus, one would expect a similar percentage of long responses for the 1.0-2.0-sec baseline duration pair at a $1.0-\mathrm{sec} s-c$ delay and the $1.0-1.0-\mathrm{sec}$ equal-duration pair at a $2-\sec s-c$ delay. However, during $s-c$ delay testing, the $1.0-2.0-\mathrm{sec}$ duration pair at a $1-\mathrm{sec}$ $s-c$ delay had a mean of $65.4 \%$ long responses, whereas the 1.0-1.0-sec equal-duration pair at the $2-\sec s-c$ delay had a mean of $38.1 \%$ long responses. There are two additional examples that give similar results. The $1.5-2.5-\mathrm{sec}$ baseline duration pair (i.e., $1.5 \mathrm{vs} .3 .5 \mathrm{sec}$ ) had a mean of $65.5 \%$ long responses, whereas the $1.5-1.5$-sec pair at a 2 -sec $s-c$ delay (i.e., 1.5 vs. $3.5 \mathrm{sec}$ ) had a mean of $48.8 \%$ long responses. The $2.0-3.0-\mathrm{sec}$ baseline duration pair (i.e., 2.0 vs. $4.0 \mathrm{sec}$ ) had a mean of $66.2 \%$ long responses, whereas the 2.0-2.0-sec pair at a 2-sec $s-c$ delay (i.e., 2.0 vs. $4.0 \mathrm{sec}$ ) had a mean of $45.6 \%$ long responses. The difference in long responding was statistically significant for each of the three comparisons $[t(7)=6.28,2.47$, and 3.26 , 
respectively]. Thus, the idea that pigeons timed the $s-c$ delay and added it to $c$ does not provide a viable explanation for the increase in long responding on equal-duration probe trials as the $s-c$ delay is increased .

There remain two explanations for the increase in the percentage of long responding on equal-duration probe trials that do not involve a subjective shortening process. One is instructional ambiguity/confusion between the dark $s-c$ delay and the dark ITI (Dorrance et al., 2000; Sherburne et al., 1998; Zentall, 1997, 1999, 2007). If pigeons confused a dark $s-c$ delay with a dark ITI, they may have expected $s$ to be presented following an extended dark $s-c$ delay instead of $c$. On these trials, pigeons may have responded on the basis of the comparison of a fresh memory of $c$ to no memory of $s$. In other words, although they can clearly differentiate $s$ from $c$, they may have responded as if no $s$ had been presented on that trial. This would have resulted in more long responses, the longer the duration of $c$ (i.e., the more that $c$ deviated from an $s$ of 0 ). In support of this possibility, during equal-duration probe testing, at the 8 -sec $s-c$ delay, an ordinal relationship existed between the mean percentage of long responding and the length of the comparison duration $(42.5 \%, 49.4 \%, 55.6 \%, 62.5 \%$, $75.6 \%, 82.5 \%$, and $92.5 \%$, for the seven equal-duration pairs from 1.0-4.0 sec, respectively). Another possibility is that during training - in addition to learning relational rules for comparison-common durations $(2.0,2.5$, and $3.0 \mathrm{sec})$ pigeons learned absolute rules for comparison-unique trials; that is, if $c$ equals 1.0 or $1.5 \mathrm{sec}$, respond "short," whereas if $c$ equals 3.5 or $4.0 \mathrm{sec}$, respond "long." At long $s-c$ delays during testing, with no memory of $s$, pigeons may have generalized the absolute rules for comparison-unique trials to the comparison-common durations $(2.0,2.5$, and $3.0 \mathrm{sec})$. Hence, the 3.0-3.0-sec pair would result in more long responses at the 8-sec $s-c$ delay than would the 2.0-2.0-sec pair (i.e., $75.6 \%$ vs. $55.6 \%$, respectively).

Another explanation for the increase in long responding on equal-duration probe trials is the explanation favored in the comparable human duration-comparison literature - that is, subjective shortening of $s$ (Wearden \& Ferrara, 1993; Wearden et al., 2007; Wearden et al., 2002). The subsequent experiments will undertake further tests to elucidate whether confusion or subjective shortening was responsible for the increase in long responding on comparison-common trials.

\section{EXPERIMENT 2}

Previous research on pigeons' duration-comparison performance has addressed the role of both absolute and relational control of discriminative responding (Dreyfus et al., 1988; Dreyfus et al., 1992; Fetterman, 1987; Fetterman \& Dreyfus, 1986; Fetterman et al., 1988). Because $c$ is the duration closest to the opportunity to gain reinforcement, it would not be surprising if pigeons might try to rely on the predictive value of this duration (Dreyfus et al., 1992). Kraemer (1990) presented pigeons with a standard duration that was immediately followed by a comparison duration (either $2 \mathrm{sec}$ of red followed by $8 \mathrm{sec}$ of green or $8 \mathrm{sec}$ of red followed by $2 \mathrm{sec}$ of green). One group of pigeons was required to discriminate the color that had been presented for the shorter duration, whereas a second group was required to discriminate the color that had been presented for the longer duration. Once the pigeons successfully learned the task, Kraemer administered probe trials in which only $s$ or $c$ was presented on a given trial. Kraemer found that following 8-sec probe trials, pigeons were as accurate as they were on normal trials in which they had received both $s$ and $c$. On the other hand, following 2-sec probe trials, pigeons' accuracy was significantly below chance. Kraemer concluded that the pigeons were not making a relational discrimination, but instead were using the presence or absence of the 8-sec duration as a discriminative cue for responding. Similar to a signal detection framework of memory for time (Gaitan \& Wixted, 2000; Wixted \& Gaitan, 2004), when faced with having to make a decision at a lengthy $s-c$ delay, such as $8 \mathrm{sec}$, the pigeon may search its memory for the remembered duration of $s$ that was presented, and when no memory of $s$ is recalled, the pigeon makes a long response. However, since the length of $c$ influences long responding in the duration-comparison procedure, it alone may influence the choice that a pigeon makes if the $s-c$ delay is confused with the ITI.

In Experiment 2, pigeons were presented with random probe trials that consisted only of $c$. It was predicted that if the 8-sec $s-c$ delay data from Experiment 1 can be explained in terms of the pigeons confusing the $s-c$ delay with the ITI, then the mean percent long responding on $c$-alone probe tests should not significantly differ from the mean percent long responding at the 8-sec $s-c$ delay testing in Experiment 1 . If there is no significant difference, this would suggest that the pigeons were responding as if they did not have a memory of $s$ being presented. However, if long responding at the 8-sec $s-c$ delay is significantly lower than on $c$-alone probe test trials, this would suggest that there was some memory for $s$, even at the 8-sec $s-c$ delay.

\section{Method}

\section{Subjects and Apparatus}

The same pigeons and apparatus from Experiment 1 were used in Experiment 2.

\section{Procedure}

$c$-alone probe testing. Following Experiment 1, each pigeon received a minimum of three sessions of 1 -sec baseline $s-c$ delay training prior to receiving five sessions of testing during which $s$ was omitted on probe trials. Each test session consisted of 140 baseline trials and an additional 28 probe trials during which only $c$ was presented. Within each block of 12 individually randomized trials, there were 5 baseline $c<s$ trials, 5 baseline $c>s$ trials, and $2 c$-alone probe trials. Baseline trials were the same as those described in Experiment 1. On $c$-alone trials, the three pecking keys were illuminated by a green light for a duration ranging from 1.0 to $4.0 \mathrm{sec}$ in 0.5 -sec increments. Following the presentation of $c$, all three keys were darkened for $0.5 \mathrm{sec}$; then, a yellow light was presented on the left and right keys. A peck to either side key on $c$-alone trials led directly to a variable-length dark ITI of 4 , 8,16 , or $32 \mathrm{sec}$. The statistical analysis consisted of planned comparisons with a rejection region of $p<.05$.

\section{Results and Discussion}

Figure 4 presents the mean percentage of long responding for the comparison durations used on comparison- 
unique $c<s$ trials (i.e., $1.0 \mathrm{sec}, 1.5 \mathrm{sec}$ ), on comparisoncommon trials (i.e., $2.0 \mathrm{sec}, 2.5 \mathrm{sec}, 3.0 \mathrm{sec}$ ), and on comparison-unique $c>s$ trials (i.e., $3.5 \mathrm{sec}, 4.0 \mathrm{sec}$ ). The data previously obtained during equal-duration pair testing at the 8-sec $s-c$ delay in Experiment 1 are represented on the left, and the data obtained during $c$-alone probe testing in Experiment 2 are presented on the right. In both experiments, the mean percentage of long responses varied across the three comparison trial types, which reflected an increase in long responding as the duration of $c$ increased. The mean percentage of long responding for both types of comparison-unique trials was very similar for the 8-sec $s-c$ delay test in Experiment 1 and the $c$-alone test in Experiment 2. However, for comparison-common trials, the mean percentage of long responses was slightly lower for the 8 -sec $s-c$ delay trials $(64.6 \%)$ than for $c$-alone trials (73.6\%). Planned comparisons revealed that long responding for comparison-unique $c<s$ trials was equivalent for the 8-sec $s-c$ delay data and the $c$-alone data $(t<1)$. It was also equivalent for the comparison-unique $c>s$ trials $(t<1)$. However, for comparison-common trials, there were significantly more long responses made for $c$-alone probe trials than for 8 -sec $s-c$ delay trials $[t(7)=2.62]$.

This result suggests that at the $8-\sec s-c$ delay, on comparison-common trials, a memory of $s$ was having an impact on long responding. Long responding was not significantly different when $c$ was a comparison-unique duration, but this might be expected since the pigeons could use an absolute rule based on $c$ alone for these trials. These results also suggest that subjective shortening, rather than confusion, may provide a better explanation of the data for comparison-common trials. If pigeons did confuse the $s-c$ delay with the ITI and subsequently responded on these tri-

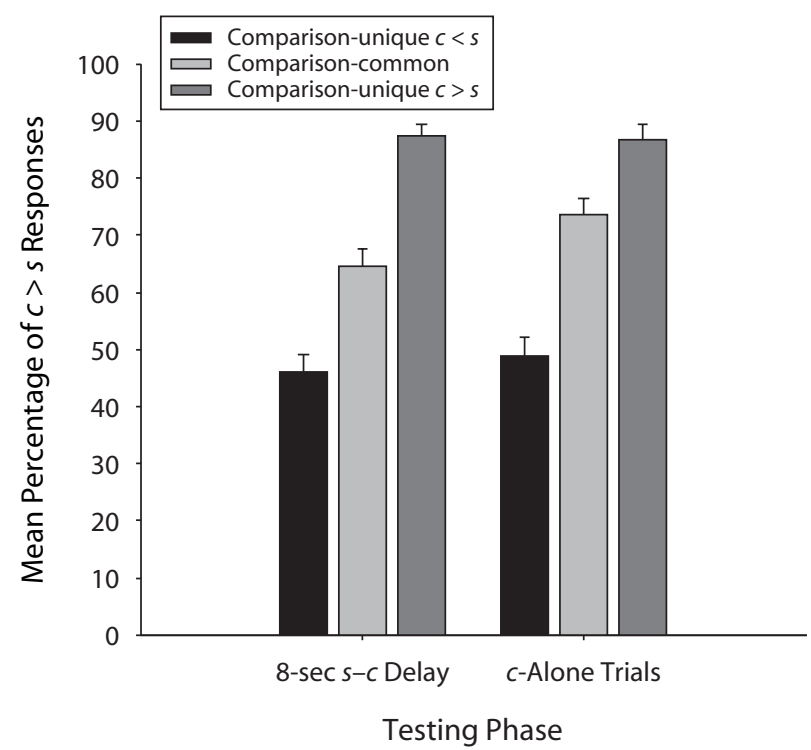

Figure 4. The mean percentage of long $(c>s)$ responding during probe testing with $c$-alone compared with 8 -sec $s-c$ delay testing for the three different types of comparison durations: $c<s$ unique (1.0 sec, $1.5 \mathrm{sec})$, comparison-common (2.0 sec, $2.5 \mathrm{sec}$, $3.0 \mathrm{sec})$, and $c>s$ unique $(3.5 \mathrm{sec}, 4.0 \mathrm{sec})$. Error bars represent the standard errors of the means. als as if no $s$ had been presented, then they would be comparing $c$ with a value of 0 . This suggests that there should have been no difference observed on comparison-common trials between $c$ alone and an 8 -sec $s-c$ delay testing. Although the $c$-alone test data might suggest that pigeons were not confusing the $s-c$ delay with the ITI, a more direct approach to ruling out instructional confusion would be preferable. Consequently, in Experiment 3, the distinctiveness of the $s-c$ delay and the ITI were manipulated.

\section{EXPERIMENT 3}

Several studies using SDMTS have demonstrated that if the ambient illumination conditions during the ITI are different from those during the DI, then the choose-short effect does not occur, and a symmetrical decline in accuracy as a function of DI length is observed for short- and longsample durations (Dorrance et al., 2000; Sherburne et al., 1998; Spetch \& Rusak, 1992). However, as was illustrated by Kelly and Spetch (2000), simply differentiating the ITI from the DI in the SDMTS procedure may not necessarily eliminate the choose-short effect. In Experiments 1 and 2 of the present article, the stimulus conditions during the $s-c$ delay and the ITI were identical. However, the results of Experiment 2 suggested that confusion may not have occurred. As was previously noted, Fetterman et al. (1988) observed an increase in long responding as a function of $s-c$ delay length, but in their study, from initial training, the entire trial presentation phase was made distinct from the ITI by presenting houselight illumination during the trial but not during the ITI. In Experiment 3, the presence of a white vertical line on all three keys throughout the entire trial presentation period was manipulated in order to make the $s-c$ delay similar to or different from the ITI.

As in the previous experiments, pigeons continued to be trained to indicate whether $c$ was either $1 \mathrm{sec}$ shorter or $1 \mathrm{sec}$ longer than $s$. The duration of $s$ consisted of a red light presented on all three keys, followed by a $1-\sec s-c$ delay, which was then immediately followed by $c$ consisting of a green light on all three keys. However, during the presentation of $s$, the $s-c$ delay, $c$, and the presentation of the choice stimuli, a white vertical line was superimposed on all three keys. Following training with the superimposed line, pigeons were tested with equal-duration pairs at $s-c$ delays of $1,2,4$, and $8 \mathrm{sec}$. During one type of test session, the white vertical lines remained present during $s$, the $s-c$ delay, $c$, and the presentation of the choice stimuli. During the other type of test session, the vertical lines remained present during $s, c$, and the presentation of the choice stimuli, but not during the $s-c$ delay. It was hypothesized that when the white vertical lines were present throughout $s$, the $s-c$ delay, $c$, and the presentation of the choice stimuli, the pigeons would not confuse the $s-c$ delay with the ITI. If the data obtained in Experiment 1 were due to confusion between the $s-c$ delay and the ITI, it was predicted that these line-present test sessions should produce $s-c$ delay functions that are different from those observed in Experiment 1. In particular, it was expected that there should be no effect of $s-c$ delay on the percentage of long responding for equal-duration pairs. However, 
on alternate test sessions in which the white vertical lines were not present during the $s-c$ delay on equal-duration test trials, it was predicted that pigeons should confuse the $s-c$ delay and ITI. If the data obtained in Experiment 1 were due to confusion between the $s-c$ delay and the ITI, it was predicted that the line-absent test sessions in Experiment 3 should produce $s-c$ delay functions similar to those obtained in Experiment 1.

\section{Method}

\section{Subjects and Apparatus}

The same pigeons and apparatus from Experiments 1 and 2 were used in this experiment.

\section{Procedure}

Discrimination training. Following Experiment 2, each pigeon received at least 1 session of $1-\sec s-c$ delay baseline training before commencing discrimination training in Experiment 3. The same discrimination training procedure used in Experiment 1 was used in the present experiment, except that a white vertical line (6-mm width) was superimposed on the center of each of the three response keys during the presentation of $s$, the $s-c$ delay, $c$, and presentation of the choice stimuli. The white vertical line stimuli also remained present for the 0.5 -sec delay between the presentation of $c$ and the choice stimuli. The duration pairs used during baseline $s-c$ delay training in Experiment 1 also remained the same, and sessions consisted of 160 trials. In order to proceed to $s-c$ delay testing, long responding on $c>s$ comparison-common trials had to exceed long responding on $c<s$ comparison-common trials by at least $25 \%$ or more in a block

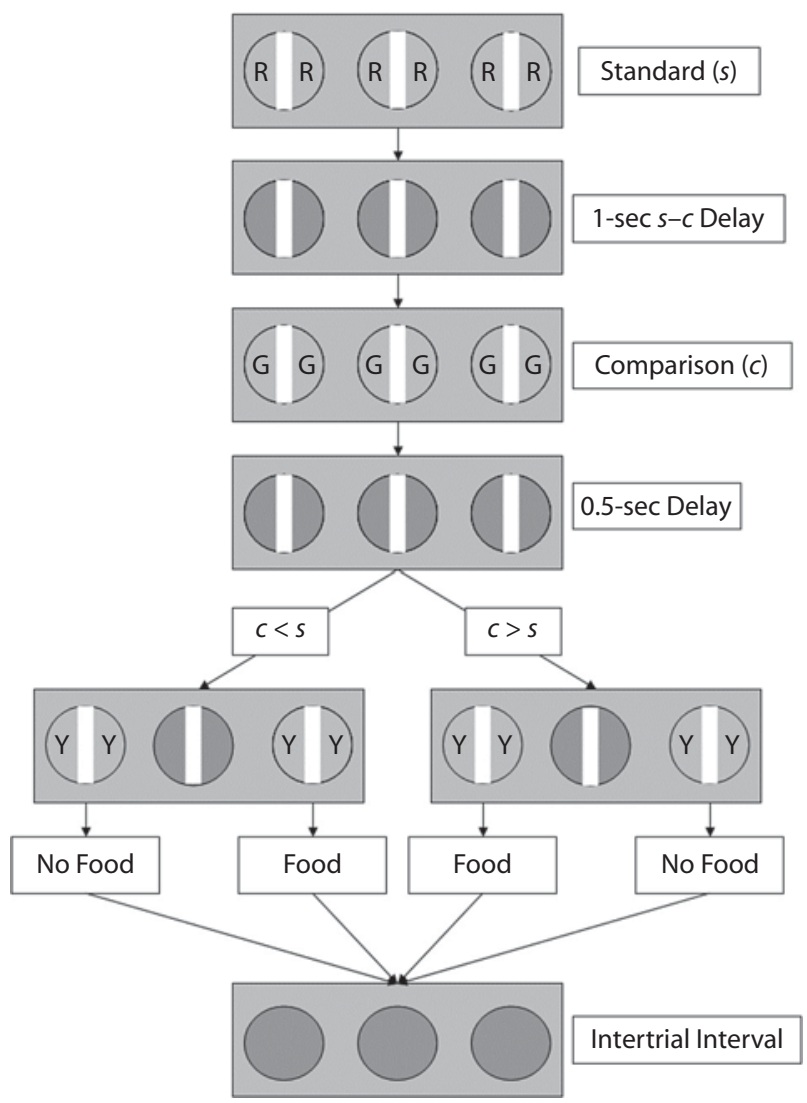

Figure 5. An illustration of the duration-comparison procedure used during line-present test sessions in Experiment 3. of 5 sessions. After 30 sessions of discrimination training, 6 of the 8 pigeons received equal-duration pair testing at various $s-c$ delays, whereas 2 birds were dropped from the study due to poor discrimination accuracy. On the last block of 5 sessions (Sessions 26 to 30), the 6 pigeons that received $s-c$ delay testing had a mean percent correct of $79.2 \%$ and $75.7 \%$ on unique and common $c<s$ trials, respectively, and a mean percent correct of $68.0 \%$ and $61.8 \%$ on unique and common $c>s$ trials, respectively. As was noted previously, this difference in accuracy reflects a bias to the $c<s$ response alternative. On the last block of 5 sessions, the mean difference in long responding between $c<s$ and $c>s$ comparison-common trials was $37.5 \%$ for the 6 birds that received testing and only $8.1 \%$ for the 2 birds that were dropped from the study.

Equal-duration probe testing. Figure 5 provides an illustration of the duration-comparison procedure used during line-present test sessions. During both baseline trials and equal-duration probe test trials, the vertical line was presented on all three keys throughout the trial. The line was never presented during the ITI. The procedure used during line-absent test sessions was identical except that during equal-duration test trials, the vertical line was removed from the keys during the $s-c$ delay. Each pigeon received 20 alternating sessions of line-present and line-absent equal-duration $s-c$ delay testing, for a total of 40 test sessions. Each test session consisted of 168 trials. Within each test session, 70 trials occurred at the 1 -sec baseline $s-c$ delay for both $c<s$ and $c>s$ duration pairs (i.e., a total of 140 baseline trials identical to training trials). Within each 6-trial block, there were 5 baseline trials and 1 equal-duration probe trial presented in an individually randomized order. Equal-duration probe trials consisted of every equal-duration pair in increments of $0.5 \mathrm{sec}$ from 1.0 to $4.0 \mathrm{sec}$, occurring at the baseline $1-\mathrm{sec} s-c$ delay as well as at three extended $s-c$ delays $(2,4$, and $8 \mathrm{sec})$, for a total of 28 probe trials per session. All statistical analyses used a rejection region of $p<.05$.

\section{Results and Discussion}

Figure 6 displays the mean percentage of long responding during line-present $s-c$ delay testing. As in Experiment 1, the mean percentage of long responding was greater for comparison-unique $c>s$ trials than for comparison-common or comparison-unique $c<s$ trials. In addition, for each comparison trial type, the mean percentage of long responding increased as the $s-c$ delay was lengthened. An ANOVA was conducted with comparison type ( $c<s$ unique, comparison-common, $c>s$ unique) and $s-c$ delay $(0,1,4$, and $8 \mathrm{sec})$ as within-subjects factors. There was a significant main effect of comparison type $[F(2,10)=28.66]$, which indicated that long responding increased as a function of the duration of $c$. Long responding also increased as a function of the length of the $s-c$ delay $[F(3,15)=4.47]$. The $s-c$ delay $\times$ comparison type interaction was not significant $[F(6,30)=1.55]$. Singlesample $t$ tests were used to assess whether the mean percentage of long responses was significantly below or above $50 \%$. For comparison-unique $c<s$, there was a + TOE at the 1- and 2-sec $s-c$ delays $[t(5)=2.57,2.01]$. For comparison-common trials, a + TOE was obtained at the $1-\sec s-c$ delay $[t(5)=3.63]$, and the - TOE at the 8 -sec $s-c$ delay was close to being significant $[t(5)=1.99$, $p=.052]$. For comparison-unique $c>s$ trials, the $-\mathrm{TOE}$ was significant for all $s-c$ delays $[t(5)=2.96,4.06,3.69$, 3.28]. As in Experiment 1, comparison-common trials demonstrated an increase in long responding as a function of $s-c$ delay length, which was characterized by a shift from a + TOE at the $1-\sec s-c$ delay to a - TOE at the 


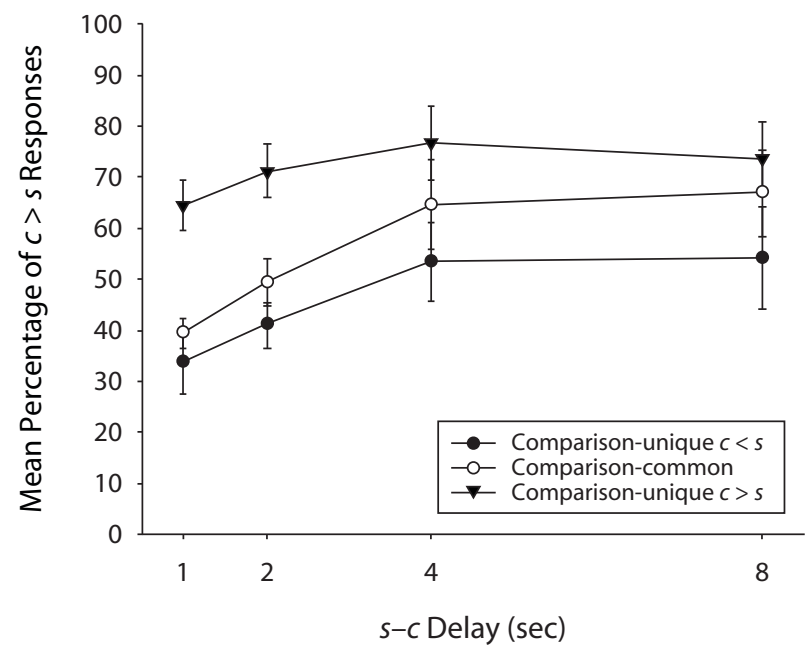

Figure 6. The mean percentage of long $(c>s)$ responding during line-present $s-c$ delay testing sessions for the three different types of comparison durations: $c<s$ unique $(1.0 \mathrm{sec}, 1.5 \mathrm{sec})$, comparison-common $(2.0 \mathrm{sec}, 2.5 \mathrm{sec}, 3.0 \mathrm{sec})$, and $c>s$ unique (3.5 sec, $4.0 \mathrm{sec})$. Error bars represent the standard errors of the means.

8 -sec $s-c$ delay. An additional ANOVA directly compared the comparison-common $s-c$ delay function obtained during line-present test sessions with the one obtained in Experiment 1. Although the main effect of the $s-c$ delay length was significant $[F(3,15)=11.66]$, there was no main effect of experiment or of $s-c$ delay $\times$ experiment interaction. This result reflects the fact that the two $s-c$ delay functions for comparison-common trials were superimposed on each other. This similarity in the $s-c$ delay function to the one obtained for comparison-common trials in Experiment 1 suggests that confusion between the

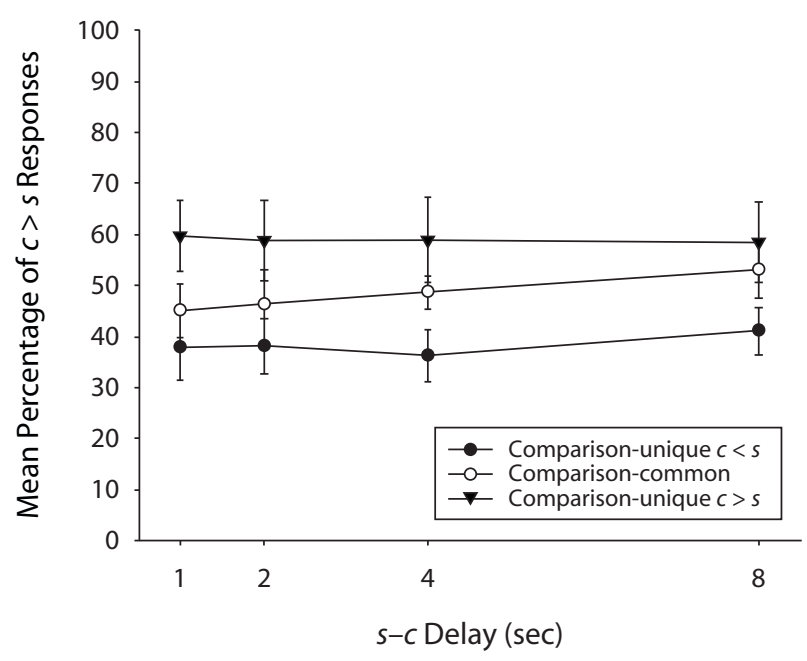

Figure 7. The mean percentage of long $(c>s)$ responding during line-absent $s-c$ delay testing sessions for the three different types of comparison durations: $c<s$ unique $(1.0 \mathrm{sec}, 1.5 \mathrm{sec})$, comparison-common (2.0 sec, $2.5 \mathrm{sec}, 3.0 \mathrm{sec})$, and $c>s$ unique $(3.5 \mathrm{sec}, 4.0 \mathrm{sec})$. Error bars represent the standard errors of the means. $s-c$ delay and the ITI was not responsible for the increase in long responding in Experiment 1.

Figure 7 presents the mean percentage of long responding during line-absent $s-c$ delay testing. Removing the line during the $s-c$ delay on equal-duration probe test trials produced $s-c$ delay functions that were very different from those obtained during line-present testing. Although the mean percentage of long responding was greater for comparison-unique $c>s$ trials than for comparisoncommon trials or comparison-unique $c<s$ trials, the length of the $s-c$ delay had no effect on long responding. An ANOVA was conducted with comparison type $(c<s$ unique, comparison-common, $c>s$ unique) and $s-c$ delay $(1,2,4$, and $8 \mathrm{sec})$ as within-subjects factors. There was a significant main effect of comparison trial type $[F(2,10)=28.66]$, which indicated that long responding was influenced by the duration of $c$. No main effect of $s-c$ delay or $s-c$ delay $\times$ comparison trial type interaction was found. The only significant deviations from chance responding were a $+\mathrm{TOE}$ at the 2 - and $4-\sec s-c$ delay on comparison-unique $c<s$ trials $[t(5)=2.22,2.76]$.

During line-absent test sessions, long responding was unaffected by the $s-c$ delay. This result is different from that observed in Experiment 1, and from the line-present $s-c$ delay data. Although it supports the claim that confusion between the $s-c$ delay and the ITI was not responsible for the data obtained in Experiment 1, it raises the question of why increases in $s-c$ delay did not continue to produce an increase in long responding when the line was absent from the $s-c$ delay. The line-absent equal-duration test trials were clearly different from the baseline training trials in Experiment 3, and the novelty of removing the line during the $s-c$ delay on these infrequent test trials appears to have resulted in the pigeons responding primarily on the basis of the duration of $c$ instead of making a relative time judgment. This disruption of performance on line-absent trials was not anticipated. As was suggested by a reviewer, it might have been better to reestablish accurate baseline performance without the lines present after the pigeons had reacquired the duration discrimination with lines present, and then conducted equal-duration test trials within the same session.

\section{GENERAL DISCUSSION}

The present experiments on duration comparison in pigeons were undertaken to determine whether the increase in long responding at extended $s-c$ delays was due to subjective shortening of $s$, or to confusion between the $s-c$ delay and the ITI. In Experiment 1, pigeons were trained in a duration-comparison procedure with a baseline 1-sec $s-c$ delay, during which the stimulus conditions in the test chamber were identical to those during the ITI. During equal-duration test trials, the mean percentage of long responses increased as the $s-c$ delay was lengthened. This increase in long responding might have been the result of pigeons comparing $s$ with the total duration of the $s-c$ delay and $c$. However, an analysis of long responding for a subset of the duration pairs produced results inconsistent with this hypothesis. At a $2-\sec s-c$ delay, equal-duration 
pairs of $1.0,1.5$, and $2.0 \mathrm{sec}$ produced significantly fewer long responses than $\operatorname{did} c>s$ training duration pairs with $c$ being equal to $2.0,2.5$, or $3.0 \mathrm{sec}$, and with a $1-\mathrm{sec} s-c$ delay. The idea that pigeons timed the $s-c$ delay and added it to $c$ does not provide a satisfactory explanation for the increase in long responding on equal-duration probe trials as the $s-c$ delay increased.

At the 8 -sec $s-c$ delay on equal-duration probe trials, long responding increased as the duration of $c$ increased. In order to determine whether there was any effect of $s$ on responding at the 8 -sec $s-c$ delay, Experiment 2 presented pigeons with test trials in which only $c$ was presented. Significantly more long responding on comparison-common trials was observed when $c$ was presented alone than on equal-duration test trials at the 8 -sec $s-c$ delay. This suggests that the presentation of $s$ on equal-duration test trials was affecting long responding even at the 8 -sec $s-c$ delay. Evidence that a memory of $s$ affected responding in this way is more consistent with the subjective shortening of $s$ than with the possibility that pigeons confused the $s-c$ delay with the ITI. Experiment 3 provided more direct evidence that pigeons were not confusing the $s-c$ delay with the ITI; it trained pigeons with a white vertical line superimposed on the three keys throughout the entire trial presentation period but not during the ITI. Pigeons were subsequently given alternating test sessions with the lines either present or absent during the $s-c$ delay on equalduration test trials. When lines were present during the $s-c$ delay but not during the ITI, the $s-c$ delay functions for comparison-common trials were identical to those obtained in Experiment 1. However, when lines were absent during both the $s-c$ delay and the ITI, there was no effect of $s-c$ delay on responding. Taken together, these results suggest that the increase in long responding as the $s-c$ delay increases on equal-duration test trials cannot be due to confusion between the $s-c$ delay and the ITI. Regardless of whether the $s-c$ delay is identical to (Experiment 1) or different from (Experiment 3 line-present test sessions) the ITI, long responding increased as a function of $s-c$ delay length on comparison-common trials. Comparisoncommon trials are particularly important, because the duration of $c$ on these trials is uninformative with respect to whether a long response will or will not be reinforced on training trials. The lack of an effect of $s-c$ delay on comparison-common trials during line-absent sessions in Experiment 3 appears to be due to generalization decrement. During training trials for this experiment, the line was always present on the keys during the $s-c$ delay. Removing the line during the $s-c$ delay for equal-duration test trials appears to have resulted in pigeons responding primarily on the basis of the duration of $c$ and not on the basis of the relative durations of $s$ and $c$.

The present data in pigeons have a number of features in common with the data obtained in humans by Wearden et al. (2007). In both species, an increase in long responses accompanied increases in $s-c$ delay on equal-duration test trials. In both species, a $+\mathrm{TOE}$ occurred at an $s-c$ delay of $1 \mathrm{sec}$ on equal-duration pair test trials. The percentage of long responses was significantly below chance (i.e., assuming that short and long responses were equally likely on these trials). However, at the longest $s-c$ delay tested (i.e., $10 \mathrm{sec}$ in humans, $8 \mathrm{sec}$ in pigeons), there was a -TOE; the percentage of long responses was significantly above chance. In pigeons, this effect was stronger in Experiment 1 than in Experiment 3, mostly because of the loss of statistical power due to subject attrition in Experiment 3. Wearden et al. (2007) argued that the increase in long responding with $s-c$ delay was not simply the result of a + TOE at the 1 -sec delay, which weakens as the $s-c$ delay increases. Instead, they suggested that a $+\mathrm{TOE}$ is present at all $s-c$ delays, but it is superimposed on a subjective shortening process that increases in strength as the $s-c$ delay increases. As was noted by Wearden et al. (2007), comparisons of data obtained from humans with those obtained from pigeons are often complicated by substantial differences in the experimental procedures. However, in the present experiments, the procedures used in pigeons were closer to those employed in Wearden et al. (2007), and the results appear to provide consistent evidence for subjective shortening in both species.

In the present experiments, pigeons clearly showed sensitivity to the predictiveness of $c$ when it was a relatively short (i.e., 1.0, $1.5 \mathrm{sec})$ or a relatively long (3.5, $4.0 \mathrm{sec})$ duration. However, in order to respond correctly to comparison-common durations $(2.0,2.5$, and $3.0 \mathrm{sec})$, the pigeons had to rely on a relational comparison. These results replicate findings previously obtained from pigeons in a duration-comparison procedure (Dreyfus et al., 1988; Dreyfus et al., 1992; Fetterman \& Dreyfus, 1986; Fetterman et al., 1988; Stubbs, Dreyfus, \& Fetterman, 1984). They suggest that pigeons rely on a mixture of absolute and relational response strategies in acquiring these temporal discriminations.

\section{AUTHOR NOTE}

This research was supported by Grant OGPOOD6378 from the Natural Sciences and Engineering Research Council of Canada to A.S. The authors thank Kelley Putzu, Kristin Lukashal, and Darlene Casemore for their animal care assistance. The data reported in this article were submitted as an MSc thesis by P.V.R. to Wilfrid Laurier University. We thank Greg Fetterman for comments on an earlier version of the article. Correspondence concerning this article should be addressed to A. Santi, Department of Psychology, Wilfrid Laurier University, Waterloo, ON, N2L 3C5 Canada (e-mail: asanti@wlu.ca).

\section{REFERENCES}

Allan, L. G. (1977). The time-order error in judgments of duration. Canadian Journal of Psychology, 31, 24-31.

Allan, L. G. (1979). The perception of time. Perception \& Psychophysics, 26, 340-354.

Dorrance, B. R., Kaiser, D. H., \& Zentall, T. R. (2000). Event-duration discrimination by pigeons: The choose-short effect may result from retention-test novelty. Animal Learning \& Behavior, 28, 344-353.

Dreyfus, L. R., Fetterman, J. G., Smith, L. D., \& Stubbs, D. A. (1988). Discrimination of temporal relations by pigeons. Journal of Experimental Psychology: Animal Behavior Processes, 14, 349-367.

Dreyfus, L. R., Fetterman, J. G., Stubbs, D. A., \& Montello, S. (1992). On discriminating temporal relations: Is it relational? Animal Learning \& Behavior, 20, 135-145.

Fetterman, J. G. (1987). Same-different comparison of duration. Animal Learning \& Behavior, 15, 403-411.

Fetterman, J. G. (2006). Duration ratio discrimination in pigeons: A criterion-setting analysis. Behavioural Processes, 71, 330-335. 
Fetterman, J. G., \& Dreyfus, L. R. (1986). Pair comparison of durations. Behavioral Processes, 12, 111-123.

Fetterman, J. G., Dreyfus, L. R., Smith, L. D., \& Stubbs, D. A. (1988). Timing and forgetting: Duration comparison with delays. Unpublished manuscript, Arizona State University.

Fetterman, J. G., Dreyfus, L. R., \& Stubbs, D. A. (1989). Discrimination of duration ratios. Journal of Experimental Psychology: Animal Behavior Processes, 15, 253-263.

Gaitan, S. C., \& WiXted, J. T. (2000). The role of "nothing" in pigeons' memory for event duration. Animal Learning \& Behavior, 28, 147-161.

Hellström, Å., \& RAmmSAYER, T. H. (2004). Effects of time-order, interstimulus interval, and feedback in duration discrimination of noise bursts in the 50- and 1000-ms ranges. Acta Psychologica, 116, 1-20.

Jamieson, D. G. (1977). Two presentation order effects. Canadian Journal of Psychology, 31, 184-194.

Jamieson, D. G., \& Petrusic, W. M. (1975a). Pairing effects and timeorder errors in duration discrimination. Perception \& Psychophysics, 18, 107-113.

Jamieson, D. G., \& Petrusic, W. M. (1975b). Presentation order effects in duration discrimination. Perception \& Psychophysics, 17, 197-202.

Jamieson, D. G., \& Petrusic, W. M. (1976). On a bias induced by the provision of feedback in psychophysical experiments. Acta Psychologica, 40, 199-206.

KelLy, R., \& SPETCH, M. L. (2000). Choice biases in delayed matchingto-sample duration with pigeons: Manipulations of ITI and delay illumination. Quarterly Journal of Experimental Psychology, 53B, 309-323.

Kraemer, P. J. (1990). Nonrelational processing of a sequential duration discrimination by pigeons. Bulletin of the Psychonomic Society, 28, 71-73.

Kraemer, P. J., Mazmanian, D. S., \& Roberts, W. A. (1985). The choose-short effect in pigeon memory for event duration: Subjective shortening versus coding models. Animal Learning \& Behavior, 13, 349-354.

Olthof, A., \& Santi, A. (2007). Pigeons (Columbia livia) associate time intervals with symbols in a touchscreen task: Evidence for ordinality but not summation. Journal of Comparative Psychology, 121, 82-94.

Santi, A., Keough, D., Gagne, S., \& Van Rooyen, P. (2007). Differential effects of empty and filled intervals on duration estimation by pigeons: Tests of an attention-sharing explanation. Behavioural Processes, 74, 176-186.

Sherburne, L. M., Zentall, T. R., \& Kaiser, D. H. (1998). Timing in pigeons: The choose-short effect may result from pigeons' "confusion" between delay and intertrial intervals. Psychonomic Bulletin \& Review, 5, 516-522.

SPETCH, M. L. (1987). Systematic errors in pigeons' memory for event duration: Interaction between training and test delay. Animal Learning \& Behavior, 15, 1-5.

SPETCH, M. L., \& RusaK, B. (1992). Temporal context effects in pigeons' memory for event duration. Learning \& Motivation, 23, 117-144.

SPETCH, M. L., \& WiLKIE, D. M. (1983). Subjective shortening: A model of pigeons' memory for event duration. Journal of Experimental Psychology: Animal Behavior Processes, 9, 14-30.

Sтотт, L. H. (1935). Time-order errors in the discrimination of short tonal durations. Journal of Experimental Psychology, 18, 741-766.

Stubbs, D. A., Dreyfus, L. R., \& Fetterman, J. G. (1984). The perception of temporal intervals. In J. Gibbon \& L. Allan (Eds.), Timing and time perception (Annals of the New York Academy of Sciences, Vol. 423, pp. 30-42). New York: New York Academy of Sciences.

Wearden, J. H., \& Ferrara, A. (1993). Subjective shortening in humans' memory for stimulus duration. Quarterly Journal of Experimental Psychology, 46B, 163-186.

Wearden, J. H., GoOdSON, G., \& Foran, K. (2007). Subjective shortening with filled and unfilled auditory and visual intervals in humans. Quarterly Journal of Experimental Psychology, 60, 1616-1628.

WeARden, J. H., PARRY, A., \& STAMP, L. (2002). Is subjective shortening in human memory unique to time representations? Quarterly Journal of Experimental Psychology, 55B, 1-25.

Wixted, J. T., \& Gaitan, S. C. (2004). Stimulus salience and asymmetric forgetting in the pigeon. Learning \& Behavior, 32, 173-182.

Woodrow, H. (1935). The effect of practice upon time-order errors in the comparison of temporal intervals. Psychological Review, 42, 127-152.

Woodrow, H., \& Stott, L. H. (1936). The effect of practice on positive time-order errors. Journal of Experimental Psychology, 19, 694-705.

Zentall, T. R. (1997). Animal memory: The role of "instructions." Learning \& Motivation, 28, 280-308.

Zentall, T. R. (1999). Support for a theory of memory for event duration must distinguish between test-trial ambiguity and actual memory loss. Journal of the Experimental Analysis of Behavior, 72, 467-472.

Zentall, T. R. (2007). Temporal discrimination learning in pigeons. Behavioural Processes, 74, 286-292.

(Manuscript received June 4, 2008; revision accepted for publication August 13, 2008.) 